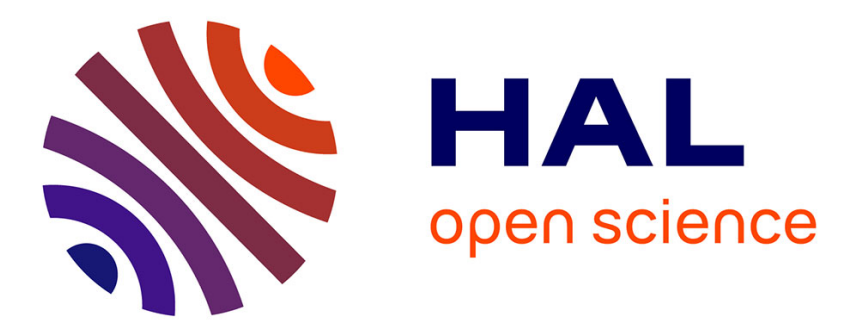

\title{
Effets d'une houle de tempête sur les mises en suspension des sédiments de fond dans le détroit du Pas-de-Calais.
}

Nicolas Guillou, Georges Chapalain

\section{- To cite this version:}

Nicolas Guillou, Georges Chapalain. Effets d'une houle de tempête sur les mises en suspension des sédiments de fond dans le détroit du Pas-de-Calais.: Comparaison de la modélisation avec les observations. European Journal of Environmental and Civil Engineering, 2010, 14 (2), pp.163-179. 10.1080/19648189.2010.9693210 . hal-01865494

\section{HAL Id: hal-01865494 \\ https://hal.science/hal-01865494}

Submitted on 15 Mar 2019

HAL is a multi-disciplinary open access archive for the deposit and dissemination of scientific research documents, whether they are published or not. The documents may come from teaching and research institutions in France or abroad, or from public or private research centers.
L'archive ouverte pluridisciplinaire $\mathbf{H A L}$, est destinée au dépôt et à la diffusion de documents scientifiques de niveau recherche, publiés ou non, émanant des établissements d'enseignement et de recherche français ou étrangers, des laboratoires publics ou privés. 


\title{
Effets d'une houle de tempête sur les mises en suspension des sédiments de fond dans le détroit du Pas-de-Calais.
}

\section{Comparaison de la modélisation avec les observations.}

\author{
Nicolas Guillou* — Georges Chapalain* \\ * Centre d'Etudes Techniques Maritimes et Fluviales, \\ Laboratoire de Génie Côtier et Environnement, Technopôle Brest-Iroise, \\ BP 5, 29280 Plouzané, France \\ nicolas.guillou@developpement-durable.gouv.fr \\ georges.chapalain@developpement-durable.gouv.fr
}

\begin{abstract}
RÉSUMÉ. Un modèle hydro-sédimentaire tridimensionnel multiclasse basé sur le couplage des codes de circulation COHERENS et de propagation de houle SWAN est appliqué à l'environnement du détroit du Pas-de-Calais et des zones côtières adjacentes au cours d'une tempête. Le modèle reproduit les mesures de houle, des courants de marée et de la concentration de sédiment en suspension totale au large de la plage de Merlimont. Les effets de la houle sur ces paramètres et les concentrations des diverses classes sédimentaires en suspension sont mis en évidence.

ABSTRACT. A three-dimensional modelling of suspended multicomponent sediment transport based on the coupling of the circulation model COHERENS and the wave propagation model SWAN is applied in the Dover Strait and its coastal adjacent areas during a storm. The model reproduces measurements of waves parameters, tidal currents and the total suspended sediment concentration off Merlimont beach. The wave effects on these parameters and the concentration of the different classes of suspended sedimentary particles are highlighted.

MOTS-CLÉS : modélisation, transport sédimentaire multiclasse, suspension, interaction houlecourant en couche limite de fond.

KEYWORDS: modelling, multicomponent sediment transport, suspension, wave-current interaction in the bottom boundary layer.
\end{abstract}




\section{Introduction}

Le domaine côtier de la Manche est considéré comme une voie importante de transfert de matières dissoutes et particulaires de l'océan Atlantique septentrional vers le sud de la mer du Nord (Salomon et al., 1993; Guéguéniat et al., 1995; Dyer et al., 1998; Velegrakis et al., 1999). L’hydrodynamisme de marée y est très actif avec des courants supérieurs à $2 \mathrm{~m} \mathrm{~s}^{-1}$ au large de la presqu'île du Cotentin ou du cap Gris-Nez (SHOM, 1973). L’hétérogénéité spatiale des sédiments de fond est globalement corrélée sur ces régimes de marée (Stride, 1963; Kenyon et al., 1970; Stride et al., 1972) avec la présence de sable fin et de vase dans les fonds de baies et les estuaires et de graviers et de cailloutis entre l'île de Wight et la presqu'île du Cotentin et dans la partie centrale du détroit du Pas-de-Calais (Vaslet et al., 1979; Larsonneur et al., 1982). A l'échelle locale des zones côtières, les faibles profondeurs favorisent également l'action de la houle sur le mouvement des sédiments de fond (Grochowski et al., 1994). Cependant, en dépit de nombreuses études numériques de l'hydrodynamisme de marée (Pingree et al., 1977; Salomon et al., 1991; Prandle et al., 1993) et des transports sédimentaires associés (Grochowski et al., 1993a; Grochowski et al., 1993b; Lafite et al., 2000), peu de modélisations ont été consacrées en Manche aux effets de la houle sur le transport sédimentaire (Velegrakis et al., 1996; Cugier, 2000; Guillou, 2007).

La présente étude vise à analyser à l'aide de codes numériques les effets d'une houle de tempête superposée au courant de marée sur les mises en suspension de particules sédimentaires de fond de différentes tailles. Ce travail se situe dans le prolongement des comparaisons modèles-mesures effectuées le long du littoral méridional du détroit du Pas-de-Calais en condition de marée seule par Guillou et al. (2006) et Guillou et al. (2009). Les résultats numériques sont comparés aux observations effectuées en condition de tempête par Chapalain et al. (1999) au large de la plage de Merlimont (Fig. 1) en octobre 1998.

L'article s’organise selon les étapes suivantes. Après une description succincte des codes numériques et des conditions de modélisation (Section 2), les résultats des simulations sont comparés aux mesures in situ et discutés afin de mettre en évidence les effets de la houle par rapport à une situation de temps calme (Section 3). Enfin, la section 4 synthétise ces résultats.

\section{Modèles numériques et conditions de simulation}

La modélisation de l'hydrodynamique est basée sur le couplage des codes de circulation tridimensionnelle (3D) COHERENS ("COupled Hydrodynamical Ecological model for RegioNals and Shelf seas”) (Luyten et al., 1999b) et de propagation spectrale de houle SWAN ("Simulating WAves Nearshore") (Booij et al., 1999). Les mises en suspension sont calculées à partir du module de transport scalaire de COHERENS adapté à la résolution de l'advection-dispersion de particules sédimentaires non-cohésives de différentes tailles (Guillou, 2007). 


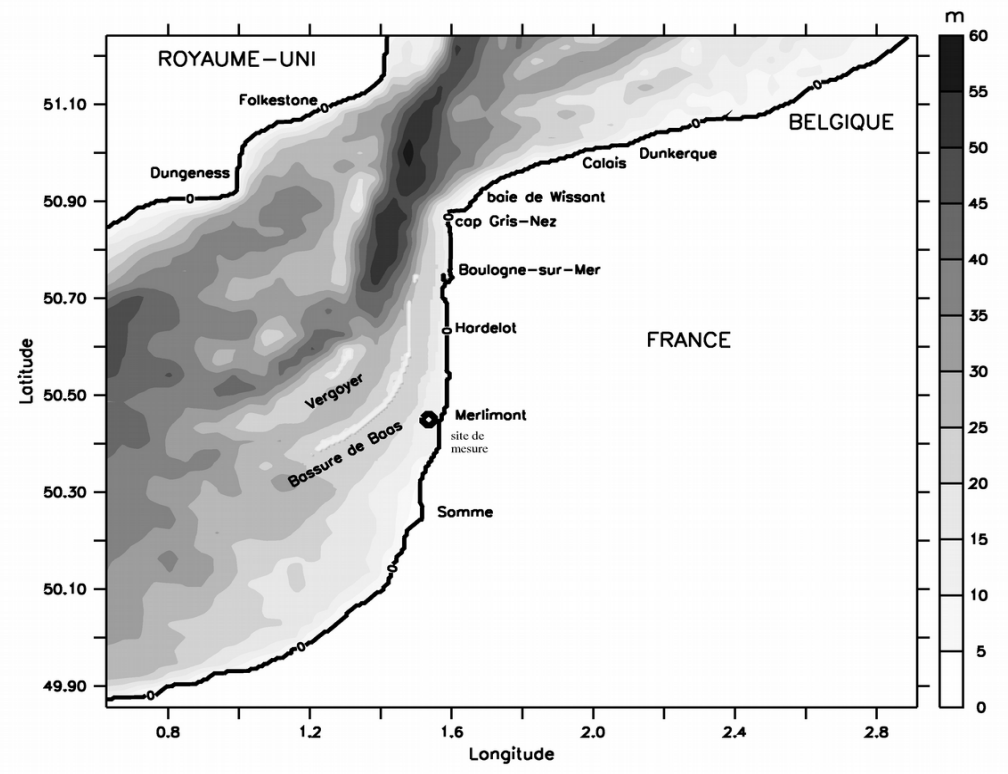

Figure 1. Bathymétrie du détroit du Pas-de-Calais et localisation du site de mesure de Merlimont.

\subsection{Modèle de circulation $3 D$}

Le noyau hydrodynamique de COHERENS résout les équations de continuité et du mouvement sous l'hypothèse d'hydrostaticité et les approximations de Boussinesq dans un système de coordonnées horizontales sphériques et verticale de type $\sigma$. Il calcule notamment l'évolution temporelle des champs 3D de vitesse moyenne, d'énergie cinétique turbulente et de viscosité. La viscosité turbulente horizontale $\nu_{\mathrm{H}}$ est paramétrée selon Smagorinsky (1963). La viscosité turbulente verticale $\nu_{\mathrm{T}}$ est calculée à partir de l'expression $\nu_{\mathrm{T}}=0,108 \mathrm{k}^{2} / \varepsilon$ où $\mathrm{k}$ est l'énergie cinétique turbulente et $\varepsilon$ est son taux de dissipation turbulente. Ces paramètres turbulent sont obtenus par la résolution d'un système à deux équations connu sous le nom de fermeture turbulente de type $\mathrm{k}-\varepsilon$ (Launder et al., 1974; Rodi, 1984). 
Les effets du vent sur la contrainte de cisaillement de surface sont paramétrés à partir de la formulation proposée par Geernaert et al. (1986). Les phénomènes d'interaction houle-courant en couche limite de fond sont pris en compte à travers un module basé sur la théorie de Grant et al. (1979) dans lequel :

- la contrainte de cisaillement totale $\tau_{\mathrm{cw}}$ est obtenue à partir de la solution analytique de l'équation du mouvement dans la couche limite de houle (Smith, 1977; Grant et al., 1979),

- la contrainte de cisaillement liée au courant $\tau_{c}$ et la vitesse de frottement associée $u_{* c}=\sqrt{\tau_{c} / \rho}$ (avec $\rho$ la densité de l'eau) intègrent les effets de rugosité apparente ressentie par le courant au-dessus de la couche limite de houle,

- les modifications sur le profil vertical de viscosité $\nu_{\mathrm{T}}$ sont imposées au niveau d'un sous-maillage de la demi-maille de fond servant à un calcul optimisé du flux de dépôt des particules sédimentaires à l'interface eau-sédiment (Guillou, 2007; Guillou et al., 2009).

Des détails supplémentaires sur le modèle et les développements entrepris sont disponibles dans Luyten et al. (1999b) et Guillou (2007).

Le modèle est implanté sur deux zones gigognes (Fig. 2) : (i) un domaine 1 couvrant la Manche orientale étendue et la partie méridionale de la mer du Nord entre les longitudes $4,000^{\circ} \mathrm{W}$ et $4,707^{\circ} \mathrm{E}$ et les latitudes $48,410^{\circ} \mathrm{N}$ et $52,837^{\circ} \mathrm{N}$ avec une résolution de $2 \mathrm{~km}$ et (ii) et un domaine 2 correspondant au détroit du Pas-de-Calais de $0,626^{\circ} \mathrm{E}$ à $2,914^{\circ} \mathrm{E}$ et de $49,856^{\circ} \mathrm{N}$ à $51,242^{\circ} \mathrm{N}$ avec une résolution de $500 \mathrm{~m}$. La discrétisation verticale comporte 11 et 14 niveaux $\sigma$ respectivement sur les domaines 1 et 2 . Les pas de temps du mode barotrope sont fixés à 20 et $10 \mathrm{~s}$ respectivement sur les domaines externes et internes. Un pas de temps de $200 \mathrm{~s}$ est adopté pour le mode barocline sur les deux domaines.

Le paramètre de rugosité est fixé sur le domaine externe à $\mathrm{z}_{0}=0,0035 \mathrm{~m}$ de la même manière que Luyten et al. (1999a) en mer du Nord. Les rugosités de grain et de formes liées aux rides sableuses sont prises en compte sur le domaine interne par l'application d'une technique originale d'interpolation des distributions granulométriques des échantillons de sédiment de fond aux différents noeuds du maillage de calcul (Leprêtre et al., 2006). Cette technique est appliquée aux 11 classes granulométriques, s'étalant des silts $(\mathrm{d}<50 \mathrm{~mm})$ aux cailloutis $(\mathrm{d}>2 \mathrm{~cm})$ et affleurements rocheux, issues des données des campagnes « RCP 378 Benthos de la Manche » (Cabioch et al., 1977) (Tableau 1 et Fig. 3). Le paramètre de rugosité est déduit des diamètres sédimentaires $\mathrm{D}_{50}$ et $\mathrm{D}_{90}$ (au-dessous desquels on retrouve respectivement 50 et $90 \%$ de la masse sédimentaire) calculés à partir de la distribution granulométrique interpolée. Les sables de diamètre médian inférieur à $800 \mu \mathrm{m}$ développent des rides (Soulsby, 1997) dont le paramètre de rugosité est 
calculé par la formule proposée par Wooding et al. (1973) à partir du modèle géométrique de Yalin (1985) de sorte que $\mathrm{z}_{0} \simeq 8 \mathrm{D}_{50}$ (Chapalain et al., 2000). Les substrats plus grossiers (i.e., $\mathrm{D}_{50} \geq 800 \mu \mathrm{m}$ ) ne présentent pas de rides et le paramètre de rugosité est pris égal à $\mathrm{z}_{0}=\mathrm{D}_{90} / 10$ (Van Rijn, 1993).

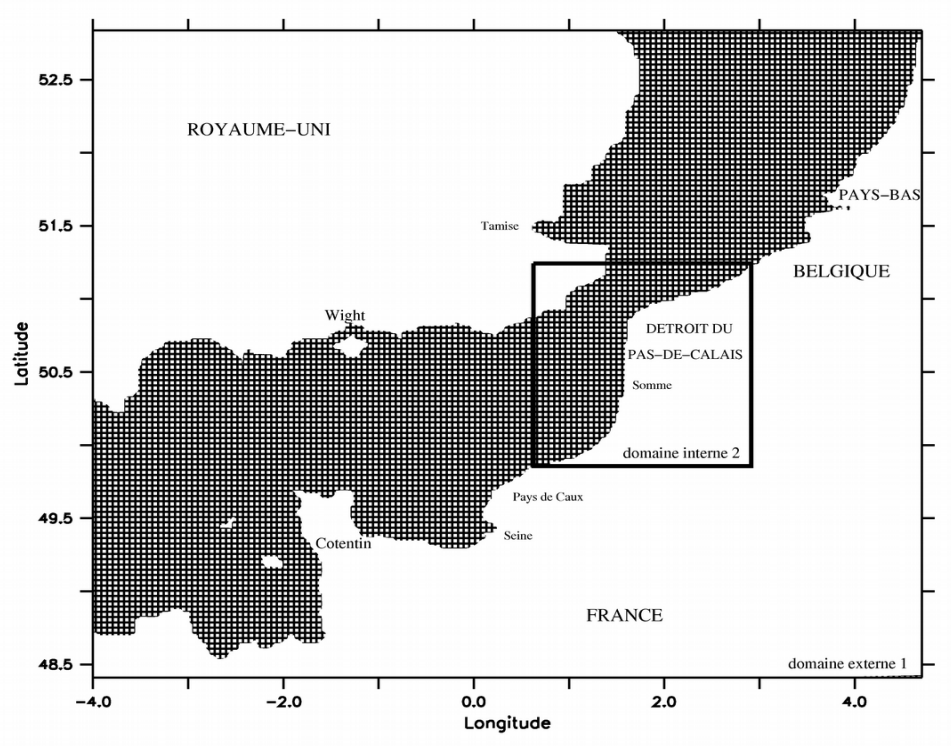

Figure 2. Domaines de calcul 1 et 2.

\begin{tabular}{|c|c|c|}
\hline Classes & Tailles des tamis & Diamètres $\mathrm{d}_{i}$ \\
\hline 1 & $0-50 \mu \mathrm{m}$ & $25 \mu \mathrm{m}$ \\
\hline 2 & $50-100 \mu \mathrm{m}$ & $75 \mu \mathrm{m}$ \\
\hline 3 & $100-200 \mu \mathrm{m}$ & $150 \mu \mathrm{m}$ \\
\hline 4 & $200-500 \mu \mathrm{m}$ & $350 \mu \mathrm{m}$ \\
\hline 5 & $0,5-1 \mathrm{~mm}$ & $750 \mu \mathrm{m}$ \\
\hline 6 & $1-2 \mathrm{~mm}$ & $1,5 \mathrm{~mm}$ \\
\hline 7 & $2-5 \mathrm{~mm}$ & $3,5 \mathrm{~mm}$ \\
\hline 8 & $0,5-1 \mathrm{~cm}$ & $7,5 \mathrm{~mm}$ \\
\hline 9 & $1-2 \mathrm{~cm}$ & $1,5 \mathrm{~cm}$ \\
\hline 10 & $2-30 \mathrm{~cm}$ & $16 \mathrm{~cm}$ \\
\hline 11 & $0,3-1 \mathrm{~m}$ & $65 \mathrm{~cm}$ \\
\hline
\end{tabular}

Tableau 1. Tailles des tamis et diamètres considérés pour chaque classe sédimentaire. 


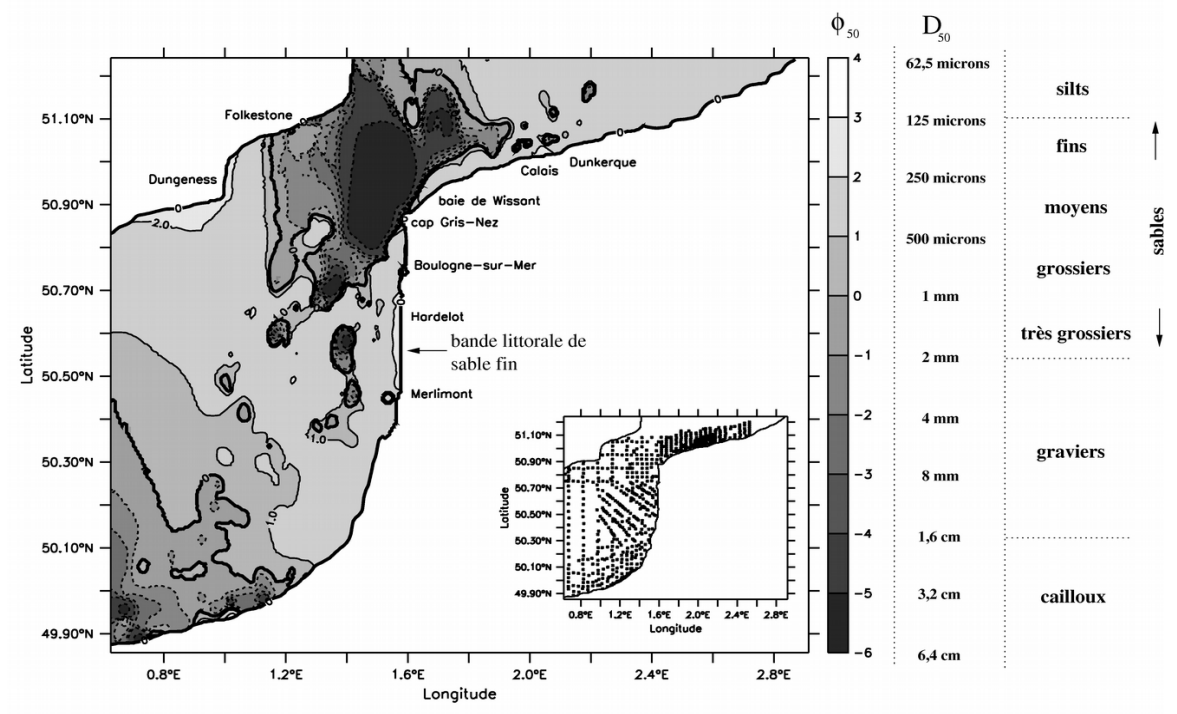

Figure 3. Répartition spatiale du diamètre médian $\phi_{50}=-\log _{2}\left(D_{50}\right)$, avec $D_{50}$ en $\mathrm{mm}$, sur le domaine 2 calculée par la technique proposée par Leprêtre et al. (2006). La Figure en bas à droite présente la répartition spatiale des échantillons des campagnes « RCP 378 Benthos de la Manche ».

L'élévation de la surface libre est imposée aux limites ouvertes du domaine externe à partir des résultats de la version bidimensionnelle de COHERENS sur le domaine épicontinental des îles britanniques (José OZER - communication personnelle). Les conditions aux frontières du domaine 2 sont la surface libre et les courants prédits sur le domaine 1. Les simulations effectuées sur le domaine interne intègrent les effets en surface des vents de la ré-analyse ERA 40 de l'ECMWF ("European Centre for Medium-Range Weather Forecasts") et les évolutions des états de mer prédits par SWAN.

\subsection{Modèle de propagation de houle}

SWAN résout l'équation d'advection-diffusion des densités spectro-angulaires de variance des vagues $\mathrm{N}_{\omega}=\mathrm{E}_{\omega} / \sigma_{\omega}$ (où $\mathrm{E}_{\omega}$ est la densité spectro-angulaire d'énergie des vagues et $\sigma_{\omega}$ est la pulsation intrinsèque) (Mei, 1983; Komen et al., 1994) et 
calcule les évolutions dérivées de la hauteur significative spectrale $\mathrm{h}_{\mathrm{m} 0}$, de la période, de la direction et de la vitesse orbitale $u_{b, r m s}$ de la houle. $h_{m 0}$ et $u_{b, r m s}$ sont obtenus à partir des expressions suivantes :

$$
\begin{aligned}
& h_{m 0}=4 \sqrt{\iint E_{\omega}\left(\sigma_{\omega}, \theta\right) d \sigma_{\omega} d \theta} \\
& u_{b, r m s}=\sqrt{2 \iint \frac{\sigma_{\omega}^{2}}{\sinh ^{2}\left(k_{\omega} H\right)} E_{\omega}\left(\sigma_{\omega}, \theta\right) d \sigma_{\omega} d \theta}
\end{aligned}
$$

où $\theta$ est la direction de propagation de la houle, $\mathrm{k}_{\omega}$ est le module du vecteur nombre d'onde des vagues défini à partir de la relation de dispersion des ondes de surface libre modifiée en présence d'un courant et $\mathrm{H}$ est la hauteur d'eau totale. La génération par le vent est basée sur le modèle de Komen et al. (1984). La dissipation des vagues par moutonnement est formulée par le modèle de Hasselmann (1974). Des détails supplémentaires sur la description des processus de génération et de dissipation de la houle pris en compte et les méthodes de résolution numérique sont disponibles dans Booij et al. (2004).

Le modèle est mis en place sur le maillage du domaine 2 (Fig. 2). Les simulations sont effectuées en mode instationnaire avec un pas de temps de $5 \mathrm{mn}$ et une discrétisation de la densité de variance des vagues en (i) 60 directions selon une résolution de $6^{\circ}$ et (ii) 30 fréquences réparties de 0,0566 à $1 \mathrm{~Hz}$ selon une progression géométrique (Lin et al., 2002).

Les conditions limites de houle correspondent à un spectre de "JONSWAP" imposé le long des frontières ouest et nord à partir des données de l'atlas numérique de houle le long des côtes françaises (Benoit et al., 2004). SWAN intègre (i) les phénomènes de génération et de dissipation par les vents de la ré-analyse ERA40 de l'ECMWF et (ii) les variations de la hauteur d'eau et des courants de marée prédits par COHERENS.

\subsection{Modèle de transport sédimentaire en suspension multiclasse}

La suspension est le seul mode de transport considéré. L'évolution spatiotemporelle de la concentration volumique de chaque classe sédimentaire en suspension $\mathrm{C}_{\mathrm{i}}$, avec $\mathrm{i} \in\left[1, \mathrm{~N}_{\mathrm{p}}\right]$ et $\mathrm{N}_{\mathrm{p}}$ le nombre de classe considéré, est régie par l'équation d'advection-diffusion des quantités scalaires de COHERENS. Le coefficient de diffusion turbulente horizontale $\lambda_{\mathrm{H}}$ est paramétré selon Smagorinsky 
(1963). Le coefficient de diffusion turbulente verticale $\lambda_{T}$ est calculé à partir de l'expression $\lambda_{T}=0,177 \mathrm{k}^{2} / \varepsilon$. La vitesse de chute des particules sédimentaires $\mathrm{w}_{\mathrm{si}}$ est obtenue à partir de l'expression proposée par Soulsby (1997).

La condition de flux imposée au voisinage du fond résulte des contributions antagonistes (i) du flux d'érosion ascendant $E_{\mathrm{i}}$ et (ii) du flux descendant de dépôt convectif $\mathrm{D}_{\mathrm{i}}$ dû à la sédimentation des particules en suspension sous l'effet de la vitesse de chute. La formulation du flux érosif est inspirée des travaux de Van Rijn (1986), Celik et al. (1988), Celik et al. (1991) et Chapalain et al. (2000). Elle se base sur l'hypothèse physique qu'en situation de source illimitée, l'écoulement érode toujours autant de sédiment du fond que sa capacité instantanée le permet. L'entraînement est toujours maximal et égale celui atteint, en régime d'équilibre, entre l'érosion et le dépôt : $E_{i}=w_{s i} i_{i}^{\text {ref }}$ avec $C_{i}^{\text {ref }}$ la concentration de référence calculée à partir de l'expression proposée par Smith et al. (1977). Le flux de dépôt est admis comme le produit de la concentration de sédiment en suspension (CSS) calculée au niveau de la première cellule verticale au-dessus du fond $C_{i}^{\text {bot }}$ par la vitesse de chute (Lick, 1982; Lavelle et al., 1984; Chapalain et al., 2000) : $D_{i}=w_{s i} C_{i}^{b o t}$.

Les pré-analyses effectuées sur le mouvement des sédiments de fond ont limité le calcul aux quatre premières classes $\left(\mathrm{N}_{\mathrm{p}}=4\right): \mathrm{d}_{1}=25 \mu \mathrm{m}, \mathrm{d}_{2}=75 \mu \mathrm{m}, \mathrm{d}_{3}=150 \mu \mathrm{m}$ et $\mathrm{d}_{4}=350 \mu \mathrm{m}$. Les transports sédimentaires sont uniquement calculés sur le sousdomaine 2 où les disponibilités des différentes classes sédimentaires sont effectivement prises en considération (Fig. 3).

\section{Applications}

\subsection{Comparaison avec les mesures à point fixe}

\subsubsection{Les observations}

Les résultats du modèle sont comparés aux mesures effectuées au large de la plage de Merlimont à la longitude $1,537^{\circ} \mathrm{E}$ et la latitude $50,450^{\circ} \mathrm{N}$ par $13,5 \mathrm{~m}$ de profondeur sur un fond de sable de diamètre médian $\mathrm{d}_{50}=256 \mu \mathrm{m}$. Le site expérimental est délimité à l'ouest par le banc de sable de la Bassure de Baas (Fig. 1). La région proche est également caractérisée par la présence au nord d'une bande littorale de sable fin (Fig. 3). L’instrumentation est déployée du 5 au 9 octobre 1998 avec à partir du 8 octobre un évènement de houle associé à un coup de vent de nord.

Le système instrumental est la Station d'Acquisition de Mesures Benthique Autonome (SAMBA). Cette structure destinée à être déployée dans un environnement soumis à de forts courants et de grosses houles (Chapalain, 2004) est équipée d'un module "haute fréquence " dédié à la mesure des vagues et des processus hydrodynamiques et hydro-sédimentaires dans la couche de fond sur une hauteur de 1,5 m à une cadence maximale de $4 \mathrm{~Hz}$. Le module comporte un capteur 
de pression (PAROSCIENTIFIC) situé à 2,17 $\mathrm{m}$ au-dessus du fond, quatre courantomètres électromagnétiques bidirectionnels de $3,8 \mathrm{~cm}$ de diamètre (MARSH-MCBIRNEY, Inc.) et quatre turbidimètres à rétro-diffusion infra-rouge (OBS-3) disposés à $0,32,0,62,0,92$ et $1,42 \mathrm{~m}$ au-dessus du fond. Des détails supplémentaires sur cette instrumentation sont disponibles dans Chapalain et al. (1999).

\subsubsection{Houle}

La Figure 4 présente les prédictions de la hauteur $\mathrm{h}_{\mathrm{m} 0}$, de la vitesse orbitale $\mathrm{u}_{\mathrm{b}, \mathrm{ms}}$ près du fond, de la direction de propagation et de la période moyenne de la houle comparées aux mesures effectuées à partir du capteur de pression positionné sur la SAMBA. L'origine des séries temporelles correspond au 5 octobre 1998 à 20:30 GMT. L'évolution de la hauteur $\mathrm{h}_{\mathrm{m} 0}$ (Fig. 4-a) est correctement simulée avec un écart inférieur à $7 \%$ au pic de tempête, le 8 octobre 1998. Le modèle approche également les phénomènes de gonflement de la houle par les courants de flot en phase avec la marée haute et opposés au sens de propagation de la houle en provenance du nord (Fig. 4-c). Les prédictions reproduisent les variations de la vitesse orbitale $\mathrm{u}_{\mathrm{b}, \mathrm{ms}}$ (Fig. 4-b) avec (i) par temps calme, des pics d'amplitude en phase avec les basses-mers et (ii) au pic de tempête du 8 octobre 1998, une augmentation d'un facteur 4 de $\mathrm{u}_{b, r m s}$. La direction de propagation (Fig. 4-c) est également modélisée. La période moyenne mesurée est plus grande que la période moyenne calculée par le modèle (Fig. 4-d). Cet écart peut s'expliquer par les difficultés associées d'une part à la mesure des hautes fréquences au niveau du capteur de pression et d'autre part à la modélisation de la génération d'une mer de vent local dans la région du site de mesure.

\subsubsection{Courants}

La Figure 5 présente les prédictions du module et de la direction des courants à 0,32 et $0,62 \mathrm{~m}$ au-dessus du fond comparées aux mesures au site expérimental de Merlimont. Les résultats numériques s'accordent avec les observations avec un retard de phase globalement inférieur à $10 \mathrm{mn}$. En période de temps calme $\left(h_{m 0}<0,8 \mathrm{~m}\right)$, le modèle reproduit l'asymétrie des courants avec une composante de flot orientée au nord de près de $25 \%$ plus importante que la composante de jusant orientée au sud. Cette asymétrie apparaît entre les deux niveaux de mesure sensiblement surestimée à $0,32 \mathrm{~m}$ au-dessus du fond en début de période de mesure. En condition de tempête $\left(h_{m 0}>1,2 \mathrm{~m}\right)$, l'atténuation des courants liée à l'augmentation du paramètre de rugosité apparente est également simulée. Au pic de tempête du 8 octobre 1998, le paramètre de rugosité apparente atteint ainsi $1,7 \mathrm{~cm}$ et la comparaison avec une simulation ne prenant pas en compte la houle montre une atténuation de l'amplitude des courants de $24 \%$. Enfin, les mesures sont caractérisées le 8 octobre 1998 par un pic d'amplitude faiblement reproduit par le 
modèle. L'existence de ce pic semble associé à un coup de vent local de quelques heures confirmé par les relevés effectués au sémaphore de Boulogne-sur-Mer et non représenté sur les données globales de l’ECMWF.
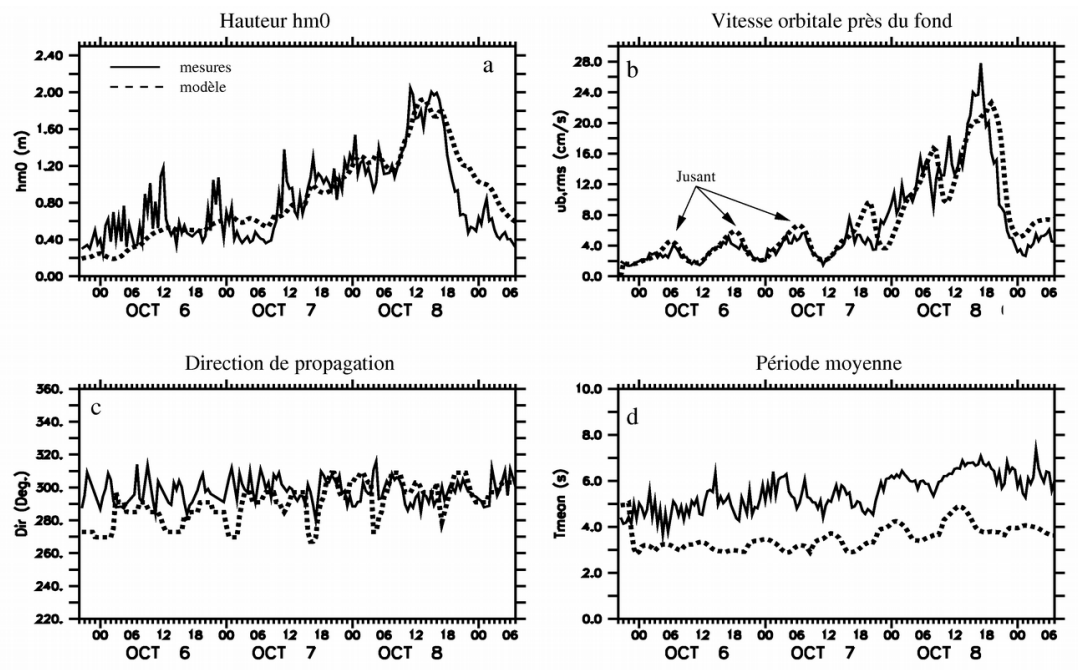

Figure 4. Chroniques de la hauteur $h_{m 0}(a)$, de la vitesse orbitale $u_{b, r m s}$ près $d u$ fond (b), de la direction de propagation repérée par rapport à la longitude dans le sens trigonométrique (c) et de la période moyenne (d), mesurées et prédites au site de Merlimont en octobre 1998.
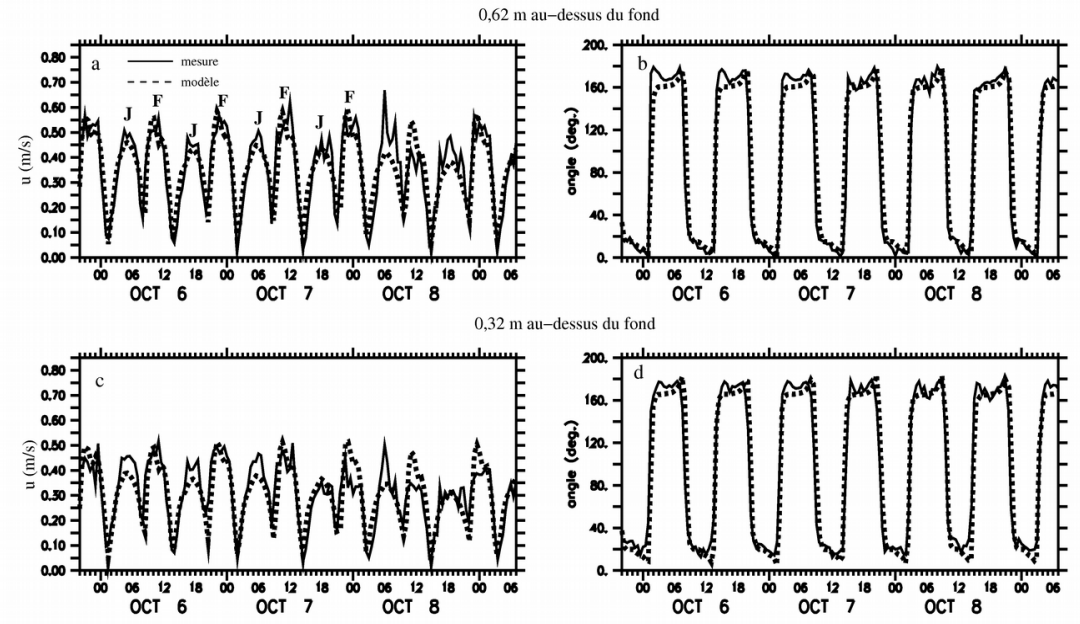

Figure 5. Chroniques du module et de la direction de la vitesse repérée par rapport au Nord dans le sens horaire mesurées et prédites à 0,32 et 0,62 m au-dessus du fond au site de Merlimont en octobre 1998. 


\subsubsection{Concentration de sédiment en suspension}

Les prédictions de la CSS totale sont comparées aux mesures effectuées à 0,32 m au-dessus du fond (Fig. 6). Par temps calme, le modèle reproduit les pics de CSS de jusant avec un écart prédictions-observations de l'intensité des concentrations qui se réduit progressivement jusqu'à atteindre $5 \%$ au deuxième pic de jusant du 7 octobre 1998. Durant la tempête, le modèle représente également correctement les amplitudes des pics de turbidité de 220 et $402 \mathrm{mg} \mathrm{l}^{-1}$ du 8 octobre 1998. Ces simulations montrent ainsi que la houle accroît d'un facteur 4 les mises en suspension à $0,32 \mathrm{~m}$ au-dessus du fond par rapport à une situation de temps calme. Cette augmentation résulte d'une exacerbation de la contrainte de cisaillement superficielle totale $\tau_{\mathrm{b}, \mathrm{cw}}$ de $2,6 \mathrm{~N} \mathrm{~m}^{-2}$ à $5 \mathrm{~N} \mathrm{~m}^{-2}$. Cette comparaison met cependant en évidence la présence de pics de CSS de flot non-identifiés au niveau des mesures. Une hypothèse envisagée est la sensibilité des capteurs OBS-3 à la nature des sédiments en suspension. Pour une même concentration, la réponse du capteur peut ainsi varier d'un facteur 200 en fonction de la taille des particules en suspension (D and A Instrument Company, 1991). Le gain diminue lorsque le diamètre des sédiments augmente.
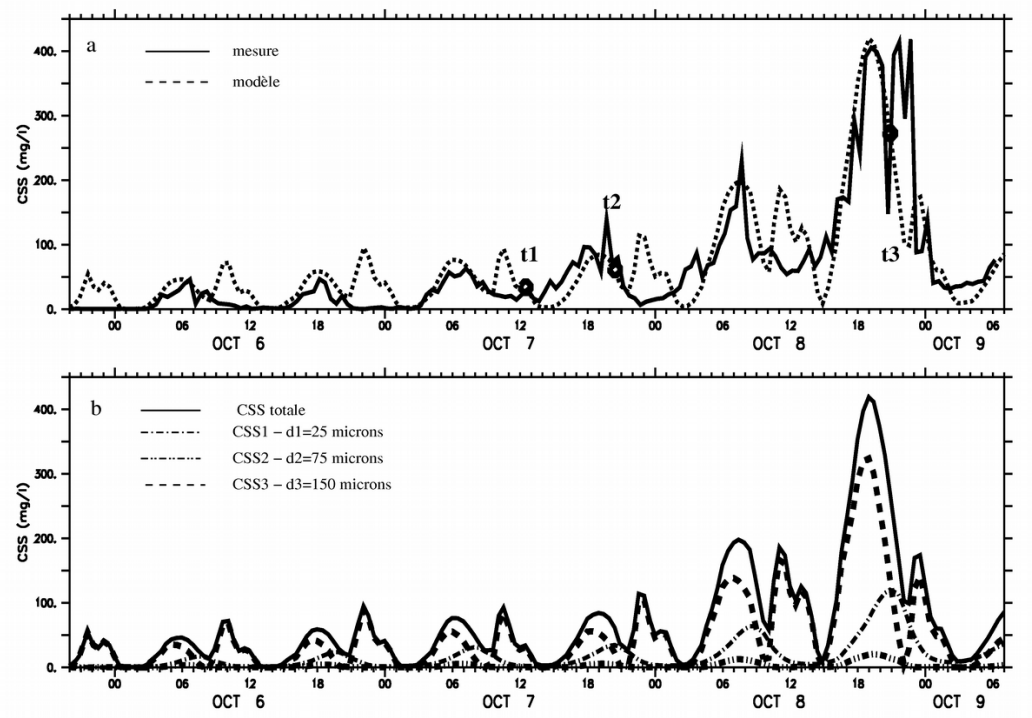

Figure 6. Chroniques de la CSS totale mesurée et prédite (a) et des concentrations de silt $\left(d_{1}=25 \mu \mathrm{m}\right)$, sable très fin $\left(d_{2}=75 \mu \mathrm{m}\right)$ et sable fin $\left(d_{3}=150 \mu \mathrm{m}\right)$ en suspension prédites (b) à 0,32 m au-dessus du fond au site de Merlimont en octobre 1998.

Cette hypothèse est confirmée par l'analyse des contributions temporelles des différentes classes au signal de CSS totale (Fig. 6). Les mises en suspension au site 
de Merlimont résultent de la contribution des trois premières classes : les silts $\left(\mathrm{d}_{1}=\right.$ $25 \mu \mathrm{m})$, les sables très fins $\left(\mathrm{d}_{2}=75 \mu \mathrm{m}\right)$ et les sables fins $\left(\mathrm{d}_{3}=150 \mu \mathrm{m}\right)$. Les pics de CSS totale en flot apparaissent liés aux mises en suspension de la classe la plus grossière de sable fin. Cette analyse décrit également les réponses (i) quart-diurnes des sables fins qui atteignent une concentration de $310 \mathrm{mg} \mathrm{l}^{-1}$ au pic de tempête et (ii) semi-diurnes des silts et sables très fins avec des maxima de CSS en fin de jusant.

\subsection{Champs synoptiques}

Les contributions des différentes classes au signal de CSS totale sont étudiées via les résultats synoptiques du modèle autour du site de mesure. Cette analyse met en évidence les mises en suspension locales des sables fins $\left(\mathrm{d}_{3}=150 \mu \mathrm{m}\right)$ en phase avec la contrainte de cisaillement locale. Les évolutions des sédiments fins sont également étudiées. La Figure 7 présente les champs synoptiques de la vitesse de frottement totale $\mathrm{u}_{* \mathrm{cw}}$ et de la concentration de silt $\left(\mathrm{d}_{1}=25 \mu \mathrm{m}\right)$ en suspension à 0,32 $\mathrm{m}$ au-dessus du fond à différents instants du cycle de marée par temps calme et en condition de tempête. Par temps calme en fin de flot (le 7 octobre à 12:30 GMT), les mises en suspension des silts restent confinées à la bande littorale de sable fin et leur contribution à la CSS totale au site de mesure est inférieure à $20 \mathrm{mg} \mathrm{l}^{-1}$. Cette situation contraste avec les mises en suspension prédites en fin de jusant (le 7 octobre à 20:30 GMT) advectées de la bande littorale de sable fin au site de mesure où leur contribution atteint $40 \mathrm{mg} \mathrm{l}^{-1}$. Les concentrations advectées sont naturellement plus importantes en condition de tempête (le 8 octobre à 21:00 GMT).

L'analyse synoptique des champs de CSS autour du site de Merlimont met ainsi en évidence les mises en suspension locales des sables fins superposées à celles des silts et sables très fins advectés en période de jusant de la bande littorale de sable fin située au nord du site de mesure.

\section{Conclusions}

Ce travail illustre, à travers une comparaison des résultats du modèle avec les observations, les effets de la houle au niveau d'une zone côtière de la Manche Orientale. Les prédictions reproduisent de manière satisfaisante les observations des caractéristiques de la houle, des courants et de la CSS totale effectuées à proximité du fond au large de la plage de Merlimont. Les interactions entre le courant de marée et la houle conduisent (i) en condition de houle modérée, à des modulations de la hauteur $h_{m 0}$ par les courants de marée et de la vitesse orbitale près du fond $u_{b, r m s}$ par l'évolution temporelle de l'élévation de la surface libre, et (ii) en période 


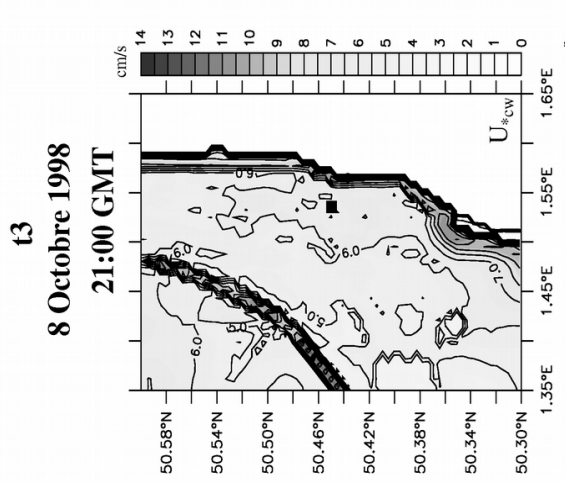

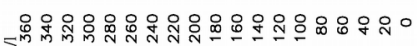
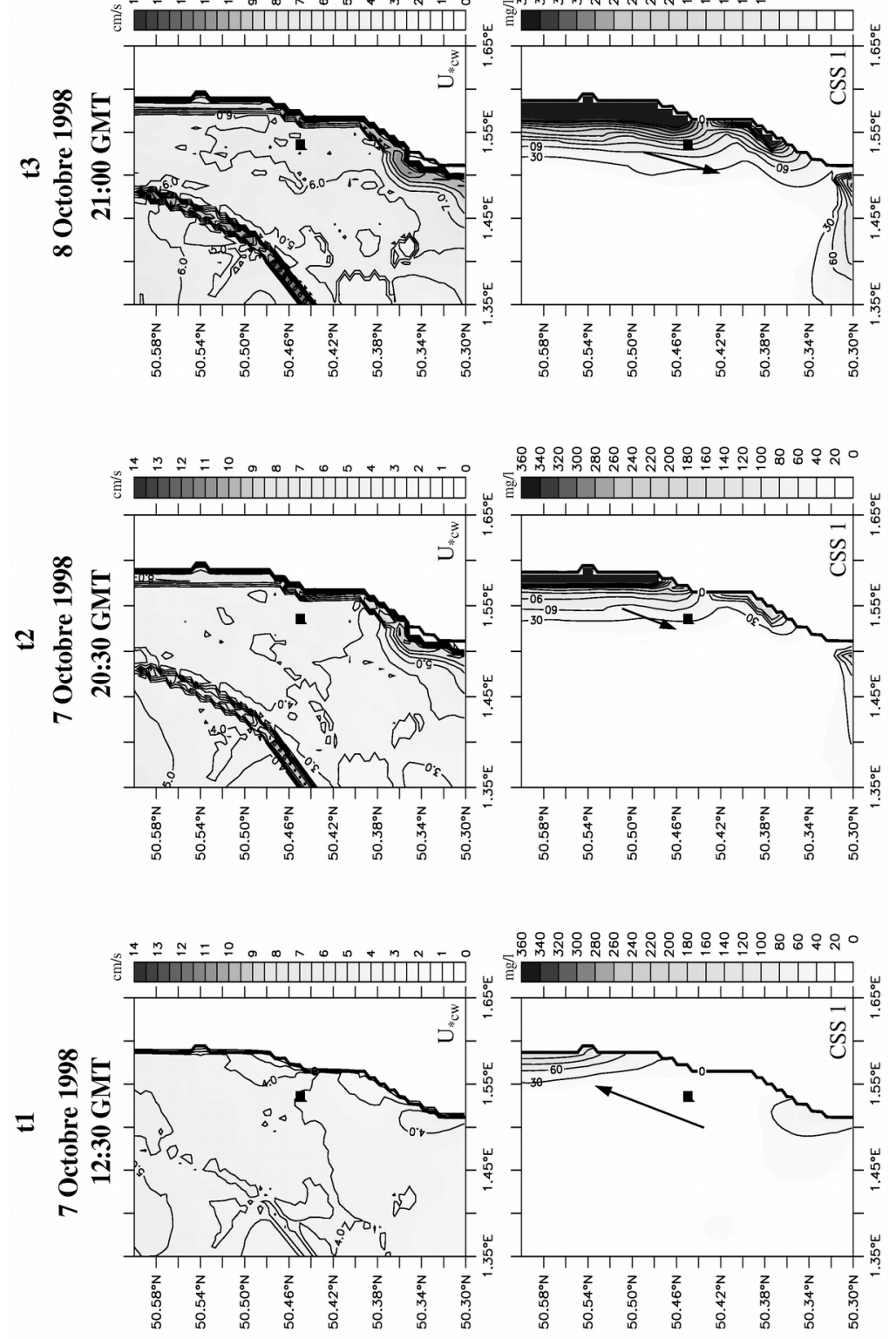

Figure 7. Champs de la vitesse de frottement totale $u_{* \mathrm{cw}}$ et de la concentration de silt $\left(d_{1}=25 \mu \mathrm{m}\right)$ en suspension à 0,32 $\mathrm{m}$ au-dessus du fond dans la région du site de mesure de Merlimont le 7 octobre 1998 à 12:30 GMT en fin de flot par temps calme, le 7 octobre 1998 à 20:30 GMT en fin de jusant par temps calme et le 8 octobre 1998 à 21:00 GMT en fin de jusant en condition de tempête. 
de tempête, à une augmentation d'un facteur 2 de la contrainte de cisaillement totale $\tau_{\mathrm{cw}}$ et d'un facteur 4 de la CSS totale dominée par la contribution des sables fins $\left(\mathrm{d}_{3}\right.$ $=150 \mu \mathrm{m})$. L'effet du phénomène d'interaction houle-courant en couche limite de fond se traduit par une réduction de l'ordre de $24 \%$ de l'amplitude des courants de marée à proximité du fond en condition de tempête. L'analyse synoptique des résultats numériques autour du site expérimental présente les contributions spécifiques des diverses classes granulométriques à la CSS totale : les sables fins mis en suspension localement, les silts et sables très fins advectés depuis la bande littorale de sable fin. Ces résultats, en lien avec ceux obtenus en condition de marée seule (Guillou et al., 2006; Guillou et al., 2009), contribuent à la mise en place d'un modèle de prédiction des flux de transport en suspension entre la Manche Orientale et la partie méridionale de la mer du Nord pour différentes conditions de marée et de houle.

\section{Remerciements}

Les auteurs tiennent à remercier José Ozer du Management Unit of the North Sea Mathematical Models (Bruxelles, Belgique) pour la mise à disposition des harmoniques de marée extraites de sa modélisation appliquée à la plateforme Atlantique, ainsi que Michel Benoit et Florence Lafon du Laboratoire National d'Hydraulique Numérique pour l'extraction des données de l'atlas numérique de houle le long des côtes françaises. Les modélisations ont été effectuées grâce aux ressources informatiques du centre de calcul pour la mer CAPARMOR. Cet article est une contribution du partenariat de recherche CETMEF-IUEM (Institut Universitaire Européen de la Mer) MEMPHYS (MEsure et Modélisation des Processus HYdrodynamiques et hydro-Sédimentaires littoraux). 


\section{Bibliographie}

Benoit M., Lafon F., «A nearshore wave atlas along the coasts of France based on the numerical modelling of wave climate over 25 years », Coastal Engineering, vol. 41, p. 714-726, 2004.

Booij N. R. C., Haagsma I. J. G., Holtthuijsen L. H., Kieftenburg A. T. M. M., Ris R. C., van der Westhuysen A. J., Zijlema M., Swan Cycle III version 40.41, User Manual, Delft University of Technology. 2004.

Booij N. R. C., Ris R. C., Holthuijsen L. H., « A third generation wave model for coastal regions, Part I, Model description and validation », Journal of Geophysical Research, vol.104, n’ C4, p. 7649-7666, 1999.

Cabioch L., Gentil F., Glacon R., Retière C., « Le macrobenthos des fonds meubles de la Manche; distribution générale et écologie ", Biology of benthic organisms, keegan, b.f. And p.o. ceidigh and p.j. caston edn, Pergamon Press, Oxford, p. 115-128, 1977.

Celik I., Rodi W., «Modelling Suspended Sediment Transport in Non-equilibrium Situations », Journal of Hydraulic Engineering, vol. 10, n 114, p. 1157-1119, 1988.

Celik I., Rodi W., « Suspended sediment-transport capacity for open channel flow », Journal of Hydraulic Engineering, vol. 2, n 117, p. 191-204, 1991.

Chapalain G., Thais L., Smaoui H., « Modeling of a tidal bottom boundary layer with suspended sediment », Hydrobiologia, vol. 414, p. 1-12, 1999.

Chapalain G., Thais L., « Tide, turbulence and suspended sediment modelling in the eastern English Channel », Coastal Engineering, vol. 41, p. 295-316, 2000.

Chapalain G., Mémoire d'Habilitation à Diriger des Recherches: Contribution à l'étude des processus hydrodynamiques et hydro-sédimentaires côtiers, HDR, Université de Bretagne Occidentale, Brest, France, 2004.

Cugier P., Développement d'un modèle numérique multicouche hétérométrique pour la simulation du transport sédimentaire en Manche; caractérisation des principaux processus., Technical Report n Rapport DPRE/SERNAT/2000-24, IRSN/LRC, 2000.

D and A Instrument Company, OBS-1 and 3, Suspended Solids and Turbidity Monitor, Technical report, Instruction Manual, 1991.

Dyer K., Moffat, T.J. « Fluxes of suspended matter in the East Anglian plume, Southern North Sea », Continental Shelf Research, vol. 18, p. 1311-1331, 1998.

Geernaert G. L., Katsaros K. B., Richter K., « Variation of the drag coefficient and its dependence on sea state », Journal of Geophysical Research, vol. 91, p. 7667-7679, 1986.

Grant W. D., Madsen O. S., « Combined wave and current interaction with a rough bottom », Journal of Geophysical Research, vol. 84, n C4, p. 1797-1808, 1979.

Grochowski N., Collins M., « Wave activity on the sea-bed of the English Channel », J. mar. bio. Ass. U.K., vol. 74, p. 739-742, 1994. 
Grochowski N. T. L., Collins M. B., Boxall S. R., Salomon J. C., « Sediment transport predictions for the English Channel, using numerical models », Journal of the Geological Society, vol. 150, p. 683-695, 1993a.

Grochowski N. T. L., Collins M. B., Boxall S. R., Salomon J. C., Breton M., Lafite R., "Sediment transport pathways in the Eastern Channel », Oceanologica Acta, vol. 16, n 5-6, p. 531-537, 1993b.

Guéguéniat P., Dubois P. B., Salomon J. C., Masson M., Cabioch L., « FLUXMANCHE radiotracer measurements: a contribution to the dynamics of the English Channel and North Sea », Journal of Marine Systems, vol. 6, p. 483-494, 1995.

Guillou N., Chapalain G., « Modélisation des mises en suspension dans le détroit du Pas-deCalais sous l'influence des courants de marée et de la houle », IXèmes Journées Nationales Génie Côtier-Génie Civil, 2006.

Guillou N., Rôles de l’hétérogénéité des sédiments de fond et des interactions houle-courant sur l'hydrodynamique et la dynamique sédimentaire en zone subtidale - applications en Manche Orientale et à la pointe de la Bretagne., $\mathrm{PhD}$ thesis, Université de Bretagne Occidentale, 2007.

Guillou N., Chapalain G., Thais L., «Three dimensional modelling of tide-induced suspended transport of seabed multicomponent sediments in the eastern English Channel », Journal of Geophysical Research - Oceans, vol. 114, C07025, 2009.

Hasselmann K., "On the spectral dissipation of ocean waves due to whitecapping ", Boundary Layer Meteor., vol. 6, n 1-2, p. 107-127, 1974.

Kenyon N., Stride A., « The tide-swept continental shelf sediments between the Shetland Isles and France », Sedimentology, vol. 14, p. 159-173, 1970.

Komen G. J., Hasselman S., Hasselman K., « On the existence of a fully developped wind-sea spectrum », Journal of Physical Oceanography, vol. 14, p. 1271-1285, 1984.

Komen G., Cavaleri L., Donelan M., Hasselmann K., Hasselmann S., Janssen P., Dynamics and Modelling of Ocean Waves., Cambridge University Press, Cambridge, 1994.

Lafite R., Shimwell S., Grochowski N., Dupont J. P., Nash L., Salomon J. C., Cabioch L., Collins M., Gao S., "Suspended particulate matter fluxes through the Straits of Dover, English Channel: observations and modelling », Oceanologica Acta, vol. 23, n 6, p. 687699, 2000.

Larsonneur C., Bouysse P., Auffret J. P., « The superficial sediments of the English Channel and its Western approaches », Sedimentology, vol. 29, p. 851-864, 1982.

Launder, B.E., Spalding, D.B., « The numerical computation of turbulent flows », Computer Methods in Applied Mechanics and Engineering, vol. 3, p. 269-289, 1974.

Lavelle J. W., Mofjeld H. O., Baker E. T., « An in situ erosion rate for fine-grained marine sediment », Journal of Geophysical Research, vol. 89, p. 6543-6552, 1984.

Leprêtre A., Chapalain G., Carpentier P., « Une méthode d’interpolation des caractéristiques granulométriques des sédiments superficiels », Bulletin de la Société Géologique de France, vol. 177, n 2, p. 89-95, 2006. 
Lick W., «Entrainment, deposition, and transport of fine-grained sediments in lakes », Hydrobiologia, vol. 91, p. 31-40, 1982.

Lin W., Sanford L. P., Suttles S. E., «Wave measurement and modeling in Chesapeake Bay », Continental Shelf Research, vol. 22, p. 2673-2686, 2002.

Luyten P. J., Jones J. E., Proctor R., Tabor A., Tett P., Wild-Aden K., Coherens: A coupled hydrodynamics-Ecological Model for Regionals and Shelf Seas - Part II Model Applications, Management Unit of the Mathematical Models of the North Sea, Belgium. 1999a.

Luyten P. J., Jones J. E., Proctor R., Tabor A., Tett P., Wild-Aden K., Coherens: A Coupled Hydrodynamics-Ecological model for REgioNals and Shelf seas - Part III Model Description (Available on CD-ROM via http://www.mumm.ac.be/coherens), Management Unit of the North Sea Mathematical Models, Belgium. 1999b.

Mei C., The applied dynamics of ocean surface waves., Wiley, New York, 1983.

Pingree R. D., Maddock L., « Tidal residuals in the English Channel », Journal of the Marine Biological Association of UK, vol. 57, p. 339-354, 1977.

Prandle D., Loch S. G., Player R., " Tidal flow through the Straits of Dover », Journal of Physical Oceanography, vol. 23, p. 23-37, 1993.

Rodi, W., "Turbulence models and their application in hydraulics ", 2nd edition, International Association for Hydraulic Research, Delft, Netherlands, 1984.

Salomon J. C., Breton M., « FLUXMANCHE’s first results in modelling hydrodynamics through the Channel and Dover Strait », (FLUXMANCHE) Hydrodynamic and Biogeochemical fluxes in the eastern Channel; Fluxes into the North Sea, vol. First Annual Progress Report, June 1990-May 1991, p. 9-14, 1991.

Salomon J. C., Breton M., « An atlas of long-term currents in the Channel », Oceanologica Acta, vol. 16, n’ 5-6, p. 439-448, 1993.

SHOM, Courants de marée de Dunkerque á Brest, Imprimerie National, Paris, 1973.

Smagorinsky J., «General circulation experiments with the primitive equations, I, the basic experiments », Monthly Weather Review, vol. 91, p. 99-164, 1963.

Smith J. D., " Modeling of sediment transport on continental shelves », The Sea, vol. 13, p. 539-577, 1977.

Smith J. D., Lean S. R. M., "Spatially averaged flow over a wavy surface », Journal of Geophysical Research, vol. 12, no 82, p. 1735-1746, 1977.

Soulsby R., Dynamics of marine sands, H. R. Wallingford, 1997.

Stride A., « Current-swept sea floors near the southern half of Great Britain », Journal of the Geological Society, London, vol. 119, p. 175-199, 1963.

Stride A., Belderson R., Kenyon N., « Longitudinal furrows and depositional sand bodies of the English Channel », Colloque sur la géologie de la Manche, Mémoires du Bureau de Recherches Géologiques et Minières, vol. 79, p. 233-240, 1972. 
Van Rijn L. C., « Mathematical modelling of suspended sediment in non-uniform flows », Journal of Hydraulic Engineering, 1986.

Van Rijn L. C., Principles of Sediment Transport in Rivers, Estuaries and Coastal Seas, vol. 6-5, Aqua Publications, Amsterdam, 1993.

Vaslet D., Larsonneur C., Auffret J. P., « Les sédiments superficiels de la MANCHE. 1/500000. Carte géologique de la marge continentale française », Ministère de l’industrie, Bureau de Recherches Géologiques et Minières, Centre National pour l'Exploitation des Océans, 1979.

Velegrakis A. F., Bishop C., Lafite R., Oikonomou E. K., Lecouturier M., Collins M. B., "Investigation of meso- and macro-scale sediment transport », Hydrodynamics biogeochemical processes and fluxes in the channel, Fluxmanche II, Final Report, MAST II, MAS2CT940089, p. 128-143, 1996.

Velegrakis A. F., Michel D., Collins M. B., Lafite R., Oikonomou E. K., Dupont J. P., Huault M. F., Lecouturier M., Salomon J. C., Bishop C., « Sources, sinks and resuspension of suspended particulate matter in the eastern English Channel », Continental Shelf Research, vol. 19, p. 1933-1957, 1999.

Wooding R.A., Bradley E.F., Marshall J.K., « Drag due to regular arrays of roughness elements of varying geometry », Boundary Layer Met., vol. 5, 285-308, 1973.

Yalin M. S., « On the determination of ripple geometry », Proc. A.S.C.E., Journ. Hydr. Div., vol. 8, n̊ 111, p. 1148-1155, 1985. 